\title{
Synthesis and physicochemical studies of fluconazole ionic liquids
}

\author{
Vaishnavi Mangrule $^{1}$, Yogesh Pore ${ }^{1 *}$, John Disouza ${ }^{2}$ \\ ${ }^{1}$ Department of Pharmaceutical Chemistry, Government College of Pharmacy, Karad, Maharashtra, 415 124, India. \\ ${ }^{2}$ Department of Pharmaceutics, Tatyasaheb Kore College of Pharmacy, Warananagar, Maharashtra, 416 113, India.
}

\section{ARTICLE INFO \\ Article history: \\ Received on: 14/06/2017 \\ Accepted on: 23/08/2017 \\ Available online: 30/11/2017 \\ Key words: \\ Fluconazole; ionic liquids; ascorbic acid; physicochemical; antifungal activity.}

\begin{abstract}
Active pharmaceutical ingredient (API) based ionic liquids (ILs) were synthesized by combination of cationic fluconazole (FLC) and anionic ascorbic acid. The solid form of the low water soluble API was converted into its liquid salt form to improve physicochemical properties and evaluate biological activity. ILs were synthesized in different molar ratios of FLC- ascorbic acid (1:1 and 1:2.5) by applying solvent evaporation method with methanol as the solvent. The analytical evidences for the formation of ILs were generated and confirmed by differential scanning calorimetry (DSC), Fourier transformation-infrared spectroscopy (FTIR) and X-ray powder diffractometry (XRPD). The solubility and dissolution of 1:1 and 1:2.5 ILs were significantly improved as compared to FLC alone, supported by decreased $\log P$ values. The improvement of physicochemical properties of IL 1:1 was found to be reliable as compared to the IL $1: 2.5$. The antifungal activity of IL 1:1 was retained against Candida albicans. However, increasing the molar ratio of ascorbic acid resulted in reduction of activity.
\end{abstract}

\section{INTRODUCTION}

Solubility is the most important physicochemical parameter to be considered in the development of pharmaceutical compounds. Various techniques have been employed over the years to improve the physicochemical parameters of active pharmaceutical ingredients (APIs) (Keramatnia et al., 2016). Most of the pharmaceutical industries depend on solid, crystalline forms of APIs, due to their improved solubility, purity, stability, manufacturing ability and also ease of handling. However, solid form of many drugs lag behind because of polymorphic conversion, low solubility and low bioavailability of crystalline solids and the tendency of amorphous form to crystallize (Stoimenovski et al., 2010). To overcome these limitations, ionic liquids (ILs) were developed to improve the physicochemical properties of pharmaceutical compounds. ILs are the salts composed of ions and are in liquid form at room

\footnotetext{
* Corresponding Author

Yogesh Pore, Department of Pharmaceutical Chemistry, Government College of Pharmacy, Karad, Maharashtra, 415 124, India, +91-02164271196; Fax: +91-02164-271196, Email: dryogeshpore@rediffmail.com
}

temperature or below $100^{\circ} \mathrm{C}$, depending on their chemical composition (Julia Romeli and Devi Wilfred, 2014; Araujo et al., 2013). The majority of the researchers world over have focused on the synthesis of ILs prepared from API. These novel compounds are third generation ILs synthesized by combination of cations and anions with improved physicochemical properties and biological activity (Pinto et al., 2013; Ferraz et al., 2011). Synthesis of ketoconazole ILs has been reported with an improved solubility in which organic acids like citric acid and tartaric acid were used as anions with ketoconazole being as a cation (Keramatnia et al., 2016). Etodolac patches were prepared by synthesizing anionic etodolac and cationic lidocaine ILs (Miwa et al., 2016). In case of flufenamic acid ILs, API was used as anion and tetrabutylammonium hydroxide, tetramethyl ammonium hydroxide, tetra butyl phosphonium hydroxide and choline hydroxide were used as cations (Julia Romeli and Devi Wilfred, 2014). However, the literature survey revealed that, API-ILs are designed in three types, the first type of ILs is via ionic bonding, where API is used as cation or anion, the second type of ILs is via covalent linkage while similar or different API are combined in one IL in which API-IL with dual activities is produced (Egorova et al., 2017). 
Fluconazole (FLC) (Fig. 1) is a triazole antifungal agent chemically known as 2-(2, 4-difluorophenyl)-1,3-bis (1H-1,2,4triazole-1-yl)-2-propanol formulated in tablet, topical and vaginal forms. It has broad spectrum of antifungal activity and is suggested for the treatment and prophylaxis of disseminated and deep organ candidiasis (Barry and Brown, 1996; Singh et al, 2011; Sharma et al., 2015). FLC has been recommended in the treatment of various superficial and systemic fungal infections. It acts by inhibiting the fungal cytochrome P450 enzyme, 14- $\alpha$ demethylase. Hence, conversion of lanosterol to ergosterol; an essential component of the fungal cytoplasmic membrane and subsequent accumulation of $14-\alpha$ demethyl sterols is prevented due to inhibition of the enzyme. The activity of FLC can be evaluated against different molds. It shows maximum inhibition against Candida albicans (Pfaller et al., 2006; Kronvall et al., 2001). However, it is a white crystalline powder and belongs to class II of Biopharmaceutical Classification System having low water solubility (Ahad et al., 2011; Bhaskar Reddy 2012). Low aqueous solubility often results in poor therapeutic outcome. Different solubility techniques were used to enhance its solubility, namely: solid dispersion (Sayeed et al., 2016), use of cyclodextrins (Yurtdas et al. 2011; Li et al. 2016; Ahad et al., 2011) and cocrystal formation with dicarboxylic acid (Kastelic et al., 2010). However, no literature reveals formation of FLC- ILs.

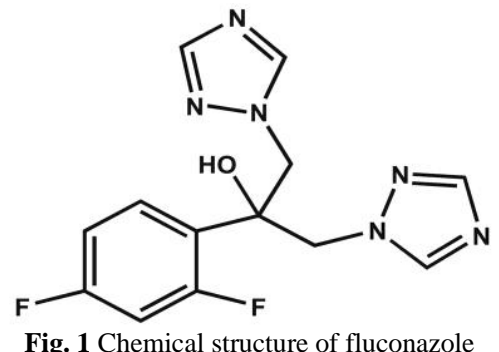

Therefore, the objective of the present work was oriented to synthesize ILs of FLC using cationic FLC and anionic ascorbic acid, based on the first type of ILs, to improve its physicochemical properties. The ILs of FLC were Synthesized using a solvent evaporation technique. The ionic hydrogen bond formation between the imidazole ring of FLC and carboxylic acid group of ascorbic acid tend to form API- based ILs. The Synthesized FLC ILs were characterized by differential scanning calorimetry (DSC), Fourier transformation-infrared spectroscopy (FTIR) and X-ray powder diffractometry (XRPD). The saturation solubility of pure FLC and corresponding ILs were Determined in distilled water, complementaory evidenced by determination of octanol / water Coefficient $(\log P)$. The dissolution or release rate studies were conducted in $0.1 \mathrm{~N} \mathrm{HCl}$. All samples were further examined for antifungal activity against Candida albicans.

\section{MATERIAL AND METHODS}

FLC was kindly gifted by Medwill Pharmaceuticals Pvt, Ltd. Satara, India. Ascorbic acid was purchased from Loba chemie
Pvt, Ltd. Mumbai, India. Candida albicans culture was purchased from NCIM resource centre, CSIR- National Chemical Laboratory, Pune, India. Analytical grade reagents and glass distilled water were used for all the experimental procedures. The substances were used without any further purification.

\section{Synthesis of FLC-ILs}

FLC-ILs was synthesized by adopting solvent evaporation method. Different molar ratios of $1: 1$ and 1:2.5 of FLC and ascorbic acid were added to $40 \mathrm{ml}$ of methanol separately and were dissolved by sonication for $20 \mathrm{~min}$. The obtained clear solutions were left for 5 days for solvent evaporation (Keramatnia et al., 2016). The products were collected and Stored in a dessicator until further analysis.

\section{Differential scanning calorimetry (DSC)}

DSC analyzer (TA Instruments SDT Q600 USA) was used to perform the thermal analysis of pure FLC, ascorbic acid, and ILs. A sample (5mg) was heated under a nitrogen atmosphere at a heating rate of $10^{\circ} \mathrm{C} / \mathrm{min}$ over the temperature range of 0 $250^{\circ} \mathrm{C}$. The DSC thermograms were then examined.

\section{Fourier transformation-infrared spectroscopy (FTIR)}

Attenuated Total Reflectance (ATR) is a sampling technique used in conjuction with infrared spectroscopy which enables samples to be examined directly in the solid or liquid state without further preparation. ATR (BRUKER- ECO- ATR ALPHA, Germany) was used for the IR analysis of all samples. The samples were directly placed on the sample pan and analyzed from 600 to $4000 \mathrm{~cm}^{-1}$ spectral range with 24 scans.

\section{X-ray powder diffractometry (XRPD)}

X-ray diffractometer (Bruker - D2 PHA-SER, Germany) with tube anode $\mathrm{Cu}$ was used to record XRPD patterns of all systems over the interval $10-90^{\circ} / 2 \theta$. The operational data were as follows: Generator tension (voltage) $30 \mathrm{kV}$, Generator current 10 $\mathrm{mA}$.

\section{Saturation solubility studies}

An excess amount of FLC and / or ILs were added to 10 $\mathrm{ml}$ distilled water in the solubility tubes. These were shaken on a orbital shaker (BTI-05) at room temperature $\left(25 \pm 2^{\circ} \mathrm{C}\right)$ until reaching equilibrium $(24 \mathrm{~h})$. A portion of the solution was withdrawn, filtered through Whatman filter paper no. 41. It was adequately diluted with distilled water. The amount of drug solubilized was determined at $261 \mathrm{~nm}$ and that of ILs at $266 \mathrm{~nm}$ by UV spectrophotometer (Shimadzu 1800 Japan).

\section{Determination of partition coefficient $(\log P)$}

The partition coefficient was determined by adding $10 \mathrm{ml}$ each of n-octanol and water in glass tubes. They were allowed to stand overnight for $24 \mathrm{~h}$ at room temperature. An accurately weighed $25 \mathrm{mg}$ of drug and ILs were added to the tubes and shaken on an incubator shaker (REMI-CIS 24 plus Incubator 
shaker, Mumbai, India) for $24 \mathrm{~h}$ at $25^{\circ} \mathrm{C}$. These mixtures were then transferred to the separating funnel and allowed to stand about $4 \mathrm{~h}$ for equilibration. Separation of aqueous and organic phases occurred. The concentrations of pure drug and ILs were analyzed spectrophotometrically (Shimadzu 1800, Japan) at $261 \mathrm{~nm}$ and 266 $\mathrm{nm}$ respectively. The formula used to calculate partition coefficient was,

Partition coefficient $(\log P)=\log ($ Octanol/C water $)$

where $\mathrm{C}$ is the concentration of drug in octanol and / or water phase (Jadhav and Pore, 2016)

\section{Release rate evaluation}

The dissolution studies of FLC and ILs were performed in triplicate in a dissolution test apparatus (ELECTROLAB-TST06L/ LX, New Mumbai, India) according to USP type II. Samples were placed in dissolution vessel containing $900 \mathrm{ml}$ of $0.1 \mathrm{~N} \mathrm{HCl}$, maintained at $37 \pm 0.5^{\circ} \mathrm{C}$ at $50 \mathrm{rpm}$ according to US FDA guidelines (US FDA website). $150 \mathrm{mg}$ of FLC and FLC-ILs was added to $900 \mathrm{ml}$ of $0.1 \mathrm{~N} \mathrm{HCl} .5 \mathrm{ml}$ of samples were withdrawn at appropriate time intervals. The volume of dissolution media was adjusted to $900 \mathrm{ml}$ by replacing each $5 \mathrm{ml}$ aliquot withdrawn with $5 \mathrm{ml}$ of fresh $0.1 \mathrm{~N} \mathrm{HCl}$. The solution was immediately filtered through Whatman filter paper No.41, adequately diluted if necessary and analyzed spectrophotometrically (Shimadzu 1800, Japan) at $260.5 \mathrm{~nm}, 245 \mathrm{~nm}$ and $244 \mathrm{~nm}$, respectively, (Auti et al., 2015).

\section{Antifungal Activity}

Antifungal activity of FLC and its ILs was determined by performing zone of inhibition using Candida albicans culture. The Sabouraud dextrose broth was used as growth media and was prepared according to the formula given. The assays were performed using Candida albicans strain with authentication number NCIM-3471. The colonies of Candida albicans were transferred (with a sterile loop) to test tube containing $5 \mathrm{ml}$ of saline solution $0.9 \%$. The resulting suspensions were stirred for 15 seconds. Two concentrations $50 \mu \mathrm{g} / \mathrm{ml}, 100 \mu \mathrm{g} / \mathrm{ml}$ were prepared by dissolving FLC in dimethyl sulfoxide and its ILs in distilled water. Agar well diffusion method was used to evaluate the zone of inhibition. The surface was inoculated by spreading a volume of the microbial inoculum over the entire agar surface. Then, a hole was punched aseptically with a sterile cork borer. The volume of $100 \mu \mathrm{L}$ of FLC and its ILs at desired concentrations were introduced into the well. The dimethyl sulfoxide was kept as control. These agar plates were freezed for 15-20 minutes in refrigerator. Then, the plates were incubated at $35^{\circ} \mathrm{C}$ for $48 \mathrm{~h}$ (Kirkpatrick et al., 1998; Leite et al., 2014; Balouiri et al., 2016).

\section{RESULTS AND DISCUSSION}

\section{Synthesis of ILs}

The white crystalline solid compound FLC (MP- $\left.138^{\circ} \mathrm{C}\right)$ with ascorbic acid (MP- $\left.194^{\circ} \mathrm{C}\right)$, after dissolving in methanol and complete solvent evaporation for 5 days appeared to be red

coloured sticky substance in all of the studied molar ratios of FLC and ascorbic acid. This indicated the synthesis of API-based ILs and conversion of a clear solution to red colored substance is a common phenomenon observed. Photographs of prepared ILs of FLC-ascorbic acid in different molar ratios are shown in Fig. 2.

(A)

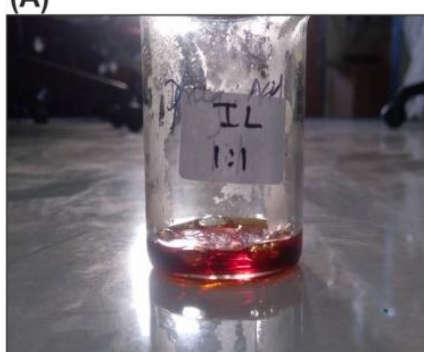

Fig. 2 Photographs of prepared FLC-ILs in different molar ratio

\section{Differential Scanning Calorimetry (DSC)}

DSC plays vital role in the investigation of interaction between API and conformers. When white crystalline FLC was converted into ILs, its melting point shifted to different temperatures or disappeared. DSC patterns of all systems are shown in Fig. 3.

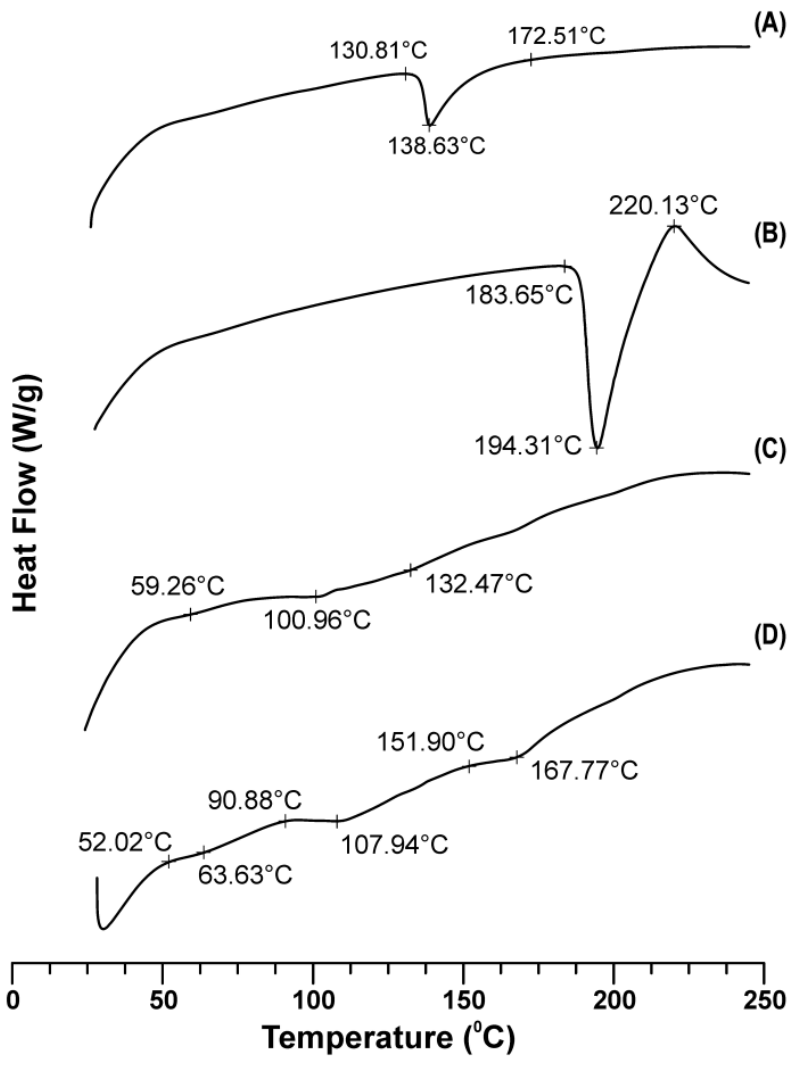

Fig. 3: DSC thermograms of FLC (A), ascorbic acid (B), IL 1:1 (C), IL 1:2.5 (D)

DSC thermogram of FLC and ascorbic acid showed sharp melting endothermic peaks at $138.63^{\circ} \mathrm{C}$ and $194.31^{\circ} \mathrm{C}$, respectively. The thermograms of ILs 1:1 and 1:2.5 did not show any peaks. This was due to conversion of solid form of drug into 
liquid salt form. The absence of melting endotherm specified their amorphous character.

\section{Fourier transformation-infrared spectroscopy (FTIR)}

The possible interaction between drug and carrier was studied by ATR-IR spectroscopy. The IR patterns of all systems are shown in Fig. 4. The principle absorption peaks of FLC were observed at 3517.47 (O-H, stretch), 3015.03 (C-H, aromatic stretch), 2877.27 (C-H aliphatic, stretch), 1616.09, $1504.18(\mathrm{C}=\mathrm{C}$, aromatic stretch), $1410.62(\mathrm{C}=\mathrm{N}$, stretch) 1269.79 (C-N stretch), $1207.93(\mathrm{C}-\mathrm{F})$ and $801.78 \mathrm{~cm}^{-1}$ ( $p$-substituted benzene). The IR spectra of ascorbic acid must show four absorption peaks of $\mathrm{O}-\mathrm{H}$ groups and lactone group. The major absorption peaks were observed at 3623.75, 3521.72, 3405.01, 3309.50 (O-H stretch), 2907.52 (C-H stretch, aliphatic) and $1749.75 \mathrm{~cm}^{-1}$ (lactone).

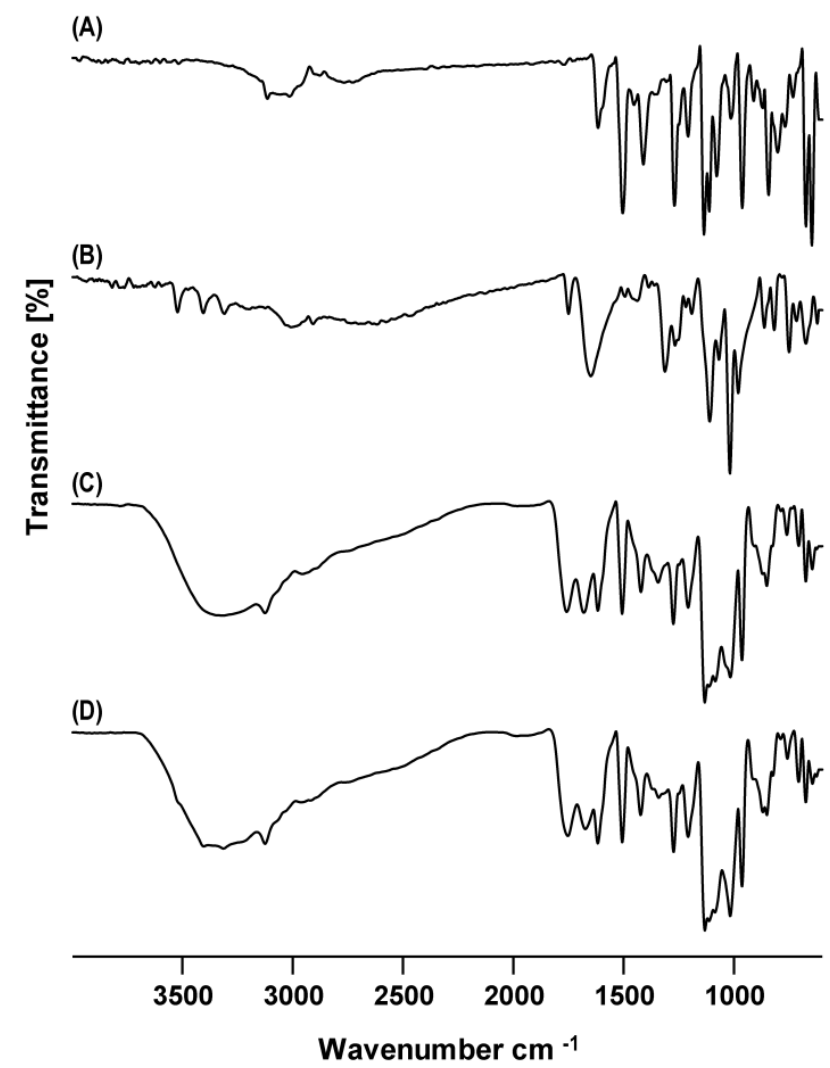

Fig. 4: FTIR spectra of FLC (A), ascorbic acid (B), IL 1:1 (C), IL 1:2.5 (D).

The functional groups of FLC (aromatic $\mathrm{C}=\mathrm{C}, \mathrm{C}=\mathrm{N}, \mathrm{C}$ $\mathrm{N}, \mathrm{C}-\mathrm{F}$ ) and lactone group of ascorbic acid were observed in both ILs and were found to be shifted. Hence, the absorption peaks of IL 1:1 were observed at 3315.19 (O-H, stretch broad), 1758.24 (lactone), $1616.94,1506.79 \quad(\mathrm{C}=\mathrm{C}$ aromatic stretch), 1421.82 (C=N, stretch), 1274.68 (C-N stretch) and at $1207.63 \mathrm{~cm}^{-1}(\mathrm{C}-\mathrm{F})$, The principle absorption peaks in IL 1:2.5 were observed at 3314.45 (O-H, stretch broad), 1752.97 (lactone), 1617.27, 1506.39 $(\mathrm{C}=\mathrm{C}$ aromatic stretch), $1422.97(\mathrm{C}=\mathrm{N}), 1273.67(\mathrm{C}-\mathrm{N})$ and at $1207.83 \mathrm{~cm}^{-1}$ (C-F). The broad peaks in IL $1: 1$ and IL 1:2.5 indicated that the heterocyclic nitrogen of FLC might be involved in the interaction with $\mathrm{OH}$ group of ascorbic acid with the transfer of proton from dissociated ascorbic acid.

\section{X-ray powder diffractometry (XRPD)}

The XRPD patterns of all samples are presented in Fig. 5. The diffractogram of FLC (Fig. 5A) exhibited $2 \theta$ values at defraction angle of 10.0016 (1125), 13.433 (483), 16.0929 (3002), 19.971 (4078), 24.9658 (1204), 28.113 (1024), 25.575 (2000) and 29.2297 (1740) while the pattern of ascorbic acid (Fig. 5B) showed the values at 10.7935 (51385), 22.8339 (107), 24.7222 (112), 30.0825 (280) and 33.8591 (146) respectively, indicating crystalline nature of both the materials. The XRPD patterns of IL 1:1 (Fig. 5C) and IL 1:2.5 (Fig 5D) showed different $2 \theta$ angles as compared to FLC and ascorbic acid. The peak intensities of both ILs were also decreased as compared to the pure drug indicating transformation of solid form of pure drug to its liquid form which were in full agreement with thermal analysis of sample.
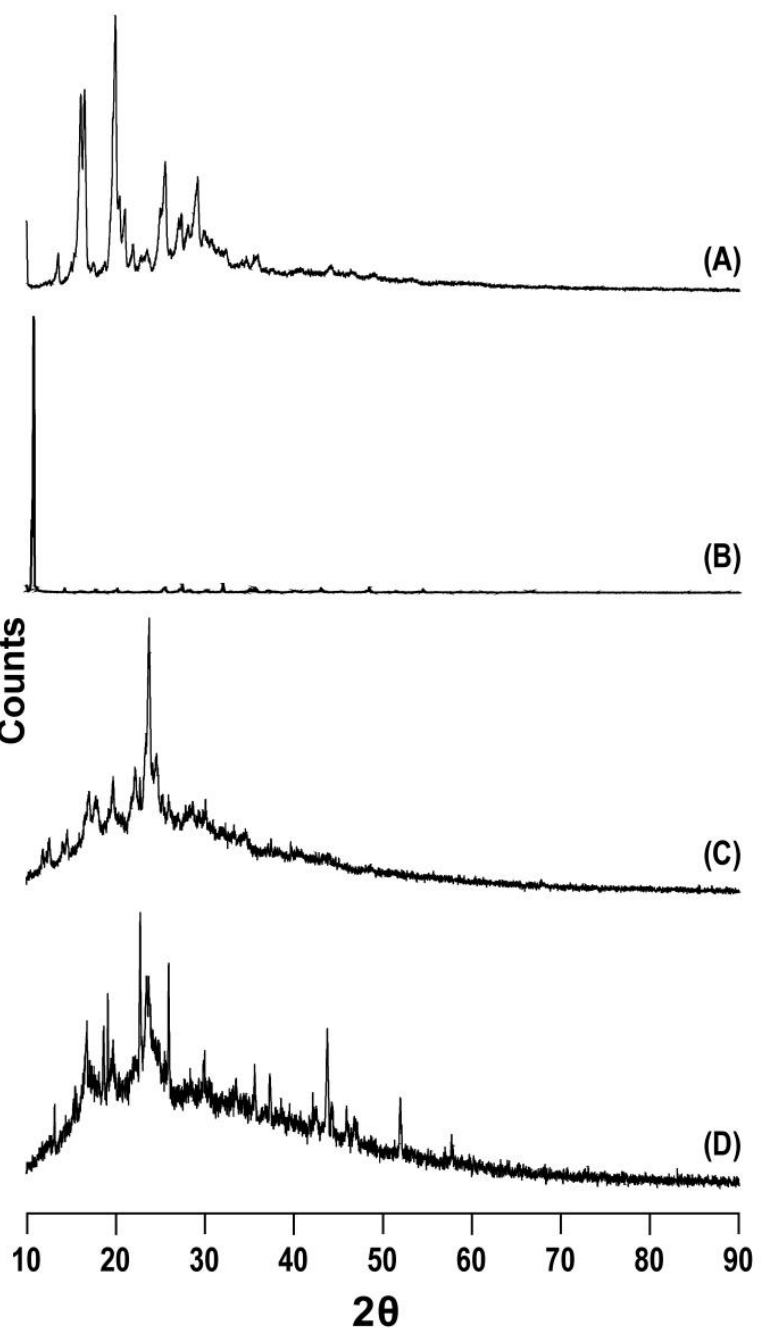

Fig. 5: XRPD patterns of FLC (A), ascorbic acid (B), IL 1:1 (C), IL 1:2.5 (D).

\section{Saturation solubility studies}

The saturation solubilities of pure FLC, 1:1 and 1:2.5 ILs was observed to be $1.476 \pm 0.152 \mathrm{mg} / \mathrm{ml}, 14.25 \pm 0.276 \mathrm{mg} / \mathrm{ml}$ 
and $14.80 \pm 0.055 \mathrm{mg} / \mathrm{ml}$ respectively. The ILs in different molar ratios $1: 1$ and 1:2.5 showed 9.65 and 10.02 fold increase in solubility as compared to pure FLC alone. There was no significant difference between the solubility of IL 1:1 and IL 1:2.5. The remarkable enhancement in solubility of FLC was attributed to its conversion into ILs with decreased crystallinity, wetting property and hydrophilicity of ascorbic acid.

\section{Determination of partition coefficient $(\log P)$}

The $\log P$ values of FLC, $1: 1$ and 1:2.5 ILs were found to be $1.60 \pm 0.01,0.516 \pm 0.025,1.37 \pm 0.025$ respectively $(p<$ $0.001)$. The comparison between $\log P$ value of pure drug and IL $1: 1$ and IL $1: 2.5$ clearly indicated that that there was significant difference between these values. The reduced $\log P$ values of both ratios of ILs compared to FLC clearly indicated enhancement in hydrophilicity of the ILs. However, the $\log P$ value of IL 1:2.5 was greater than IL 1:1 suggesting optimum amount of ascorbic acid was required to improve the physicochemical properties of FLC.

\section{Release rate evaluation}

The FLC release profile and its ILs is reported in Fig. 6. The release rate profiles were given as the percentage of drug dissolved (vs.) time. The \% dissolution efficiency (DE) at $5 \mathrm{~min}$ and 10 min was evaluated (Table 1). The dissolution rate of IL 1:1 and IL 1:2.5 was greater than that of pure FLC. A significant difference between the pure FLC and both ILs ( $p<0.001$ ) was observed. The conversion of solid form of the drug into liquid form at very low melting point by combination of cation and anion enhanced dissolution profile of ILs.

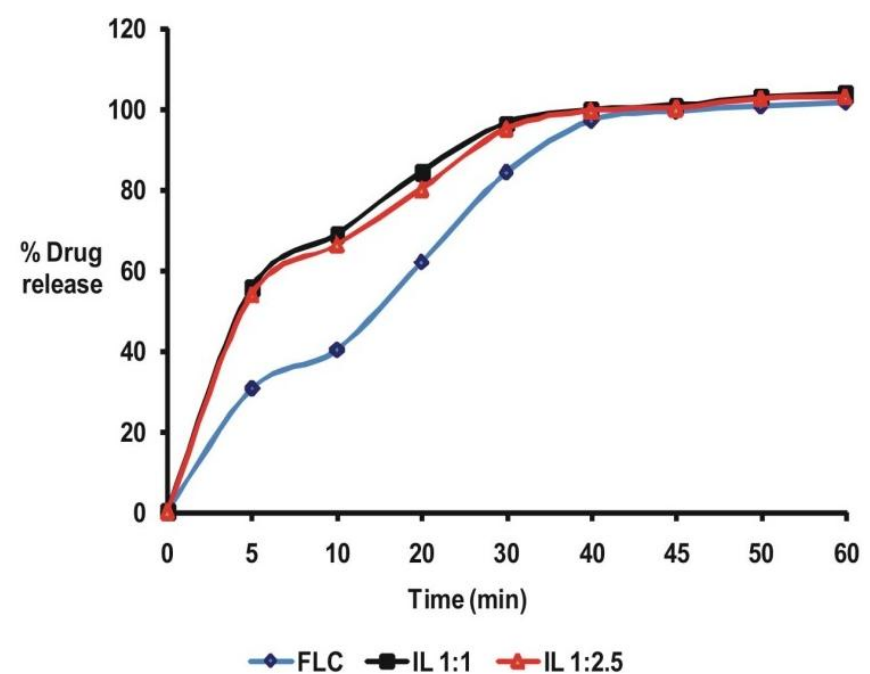

Fig. 6: The dissolution curves of FLC and ILs in $0.1 \mathrm{~N} \mathrm{HCl}$ at $37 \pm 0.5^{\circ} \mathrm{C}$ FLC: fluconazole; IL: ionic liquids.

\section{Antifungal activity}

The antifungal activity of different concentrations of FLC and ILs against Candida albicans were assessed quantitatively by measuring the diameter of zone of inhibition (Fig 7). The assay was carried out in triplicate. The results were expressed as Mean \pm SD and were given in Table 2. The results of antifungal activity of pure drug FLC and 1:1, 1:2.5 ILs were promising and showed no significant difference between FLC and $1: 1 \mathrm{IL}$ at both the concentrations of $50 \mu \mathrm{g} / \mathrm{ml}$ and $100 \mu \mathrm{g} / \mathrm{ml}$. It indicated that activity of IL $1: 1$ was retained. In case of IL 1:2.5, the activity was decreased as compared to pure drug at $100 \mu \mathrm{g} / \mathrm{ml}$ concentration while it did not show any results at $50 \mu \mathrm{g} / \mathrm{ml}$ concentration. Therefore, the results clearly specified that increase in ascorbic acid concentration led to decrease in antifungal activity.

Table 1: The $\%$ drug release and $\%$ dissolution efficiency of pure FLC, ILs 1:1 and $1: 2.5$ in $0.1 \mathrm{~N} \mathrm{HCl}$ at $37 \pm 0.5$.

\begin{tabular}{cccccc}
\hline \multirow{2}{*}{ System } & \multicolumn{3}{c}{ \% DR* } & \multicolumn{2}{c}{ \% DE* } \\
\cline { 2 - 6 } & $\mathbf{5}$ min & $\mathbf{1 0}$ min & $\mathbf{3 0}$ min & $\mathbf{5 ~ m i n}$ & $\mathbf{1 0 ~ m i n}$ \\
\hline FLC & $30.6 \pm 0.30$ & $40.2 \pm 0.60$ & $84.2 \pm 0.69$ & $15.41 \pm 0.10$ & $25.33 \pm 0.15$ \\
IL $1: 1$ & $55.54 \pm 1.00$ & $68.92 \pm 0.99$ & $96.44 \pm 0.76$ & $27.78 \pm 0.53^{\mathrm{a}}$ & $44.98 \pm 0.60^{\mathrm{a}}$ \\
IL $1: 2.5$ & $53.9 \pm 0.17$ & $66.32 \pm 0.28$ & $95.02 \pm 0.39$ & $26.95 \pm 0.087^{\mathrm{b}}$ & $43.53 \pm 0.08^{\mathrm{b}, \mathrm{c}}$ \\
\hline
\end{tabular}

* Values expressed in mean \pm S.D. $(n=3)$; S.D.: standarad deviation; FLC: fluconazole; IL: ionic liquids; \%DR: \%drug release; \%DE: dissolution efficiency; ${ }^{\text {a }}$ : FLC vs IL $1: 1$ significant difference $(p<0.001)$; ${ }^{\text {b: }}$ : FLC vs IL 1:2.5 significant difference $(p<0.001) ;{ }^{c}$ : IL $1: 1$ vs IL 1:2.5 no significant difference $(p>0.05)$.

Table 2: Antifungal activity (zone of inhibition, mm) of FLC and its ILs in different molar ratios against Candida albicans

\begin{tabular}{cccc}
\hline $\begin{array}{c}\text { Concentration } \\
\boldsymbol{\mu g} / \mathbf{m l}\end{array}$ & \multicolumn{3}{c}{ Zone of Inhibition ${ }^{*}, \mathbf{m m}$} \\
\cline { 2 - 4 } & FLC & IL 1:1 & IL 1:2.5 \\
\hline 50 & $26 \pm 1.5$ & $28 \pm 2.0$ & NR \\
100 & $28 \pm 1.5$ & $31 \pm 2.0$ & $17 \pm 1.5$ \\
\hline FLC: & &
\end{tabular}

FLC: fluconazole; IL: ionic liquids; NR: No Result; ${ }^{*}$ Values are expressed in mean \pm S.D.; S.D. standard deviation; where $n=3$; FLC vs IL $1: 1-$ No significant difference $(p>0.05)$; FLC vs IL 1:2.5 significant difference $(p<$ $0.01)$; IL 1:1 vs IL 1:2.5 - significant difference $(p<0.001)$

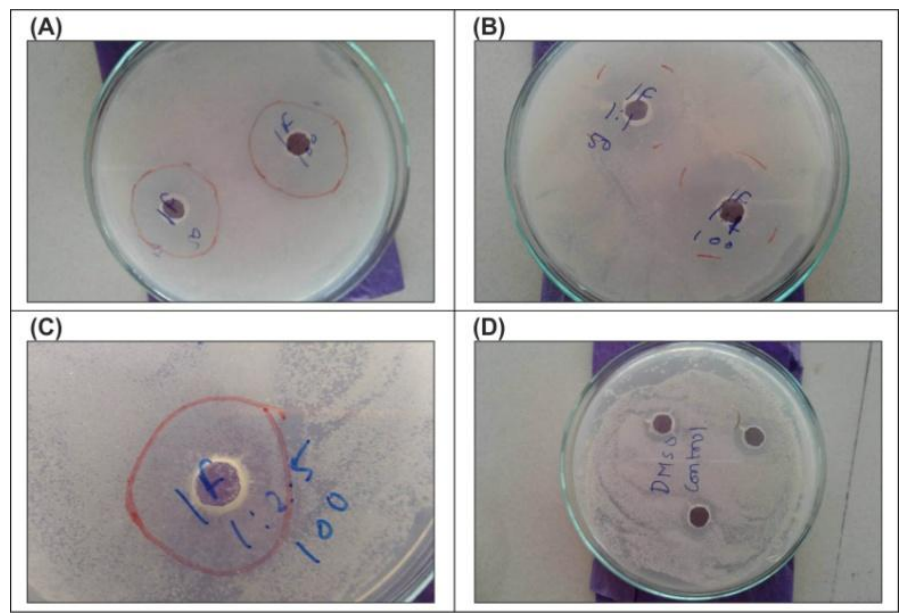

Fig. 7: Photographs of antifungal activity of FLC (A), IL 1:1 (B), IL 1:2.5 (C), DMSO control (D).

\section{CONCLUSION}

The present work demonstrated successful synthesis of ILs of cation FLC with anion ascorbic acid by solvent evaporation technique, resulting in remarkable enhancement of their physicochemical properties as compared to pure FLC. The ionic hydrogen bond formation between the imidazole ring of FLC and 
carboxylic acid group of ascorbic acid tend to form API- based ILs. The analytical techniques confirmed the formation of ILs in solid state.

The antifungal activity of synthesized ILs was retained, however, as the ratio of ascorbic acid increased the antifungal activity decreased indicating necessity of optimum ratio of FLC and ascorbic acid to form ILs. It could be concluded that formation of ILs of FLC could be an alternative and useful approach to improve the physicochemical properties of FLC.

\section{ACKNOWLEDGEMENTS}

The authors are thankful to the Principal, Government College of Pharmacy, Karad, Maharashtra, India for providing necessary laboratory facilities to carry out the research work. The authors are thankful to Shivaji University, Kolhapur, Maharashtra, India for providing analytical facilities to perform characterization studies. The gift sample of FLC provided by the Medwill Pharmaceutical Pvt. Ltd, Satara, Maharashtra, India are gratefully acknowledged. The Principal and Head of Microbiology Department, Sant Gadage Maharaj (SGM) College, Karad, Maharashtra, India are deeply appreciated for providing facilities to perform antifungal activities.

\section{Financial support and sponsorship: Nil.}

Conflict of Interests: There are no conflicts of interest.

\section{REFERENCES}

Ahad HA, Sreeramulu J, Padmaja BS, Reddy MN, Prakash PG. Preparation of fluconazole $\beta$-cyclodextrin complex ocuserts: in vitro and in vivo evaluation. International Scholarly Research Notices, 2011; DOI: 10.5402/2011/237501,1- 8 pages.

Araujo J, Florindo C, Pereiro AB, Vierira NS, Matias AA, Catarina DM, Rebelo LP,Marrucho IM. Cholinium based ionic liquids with pharmaceutically active anions. The Royal Society of Chemistry. 2014; 4: 28126-28132.

Auti SD, Jadhav SL, Gadhave MV. Dissolution method development of fluconazole in fluconazole tablets dosage form. PharmaTutor, 2015; 3(5): 29-35.

Balouiri M, Sadiki M, Ibnsouda SK. Methods for in vitro evaluating antimicrobial activity: A review. Journal of Pharmaceutical Analysis, 2016; 6: 71-79.

Barry AL, Brown SD. Fluconazole disk diffusion procedure for determining susceptibility of Candida species. Journal of Clinical Microbiology, 1996; 34(9): 2154-2157.

Bhaskar Reddy CM. Spectrophotometric estimation of fluconazole in pure drug and pharmaceutical formulation. International Journal of Scientific and Engineering Research, 2012; 3(9): 1-7.

Egorova KS, Gordeev EG, Ananikov VP. Biological activity of ionic liquids and their application in pharmaceutics and medicine. Chemical Reviews, 2017; 117(10): 7132-7189.

Ferraz R, Branco LC, Prudencio C, Noronha JP, Petrovski Z. Ionic liquids as active pharmaceutical ingredients.ChemMedChem. 2011; 6(6):975-985.

Jadhav P, Pore Y. Physicochemical, thermodynamic and analytical studies on binary and ternary inclusion complexes of bosentan with hydroxypropyl- $\beta$-cyclodextrin. Bulletin Faculty of Pharmacy, Cairo University, 2016; 55: 147-154.
Kastelic J, Hodnik Z, Sket P, Plavec J, Lah N, Leban I, Pajk M, Odon P, Kikelj D. Fluconazole cocrystals with dicarboxylic acids. Crystal Growth and Design, 2010; 10(11): 4943-4953.

Keramatnia F, Jouyban A, Valizadeh H, Delazar A. Ketoconazole ionic liquids with citric acid and tartaric acid: synthesis, characterization and solubility study. Fluid Phase Equilibria. 2016; 425:108-113.

Kirkpatrick WR, Turner TM, Fothergill AW, Mccarthy WR, Redding SW, Rinaldi MG, Patterson TF. Fluconazole disk diffusion susceptibility testing of Candida species. Journal of Clinical Microbiology, 1998; 36(11), 3429-3432.

Kronvall G, Karlsson I. Fluconazole and voriconazole multidisc testing of Candida species for disk test, calibration and MIC estimation. Journal of Clinical Microbiology, 2001; 39(4): 1422-1428.

Leite MC, Bezerra AP, Sousa JP, Guerra FQ, Lima EO. Evaluation of antifungal activity and mechanism of action of citral against Candida albicans. Evidence based Complementary and Alternative Medicine, 2014, DOI: 10.1155/2014/378280: 1-9.

Li J, Zhang S, Zhou Y, Guan S, Zhang L. Inclusion complexes of fluconazole with $\beta$-cyclodextrin and 2-hydroxypropyl- $\beta$-cyclodextrin in aqueous solution: preparation, characterization and a structural insight. 2016; 84: 209-2017.

Miwa Y, Hamamoto H, Ishida T. Lidocaine self sacrificially improves the skin permeation of the acidic and poorly water soluble drug etodolac via its transformation into an ionic liquid. European Journal of Pharmaceutics and Biopharmaceutics. 2016; 102: 92-100.

Pfaller MA, Diekema DJ, Sheehen DJ. Interpretive breakpoints for fluconazole and Candida revisited: a blueprint for the future of antifungal susceptibility testing. Clinical Microbiology Reviews, 2006; 19 (2): 435-447.

Pinto PC, Ribeiro DM, Azevedo AM, Justina VD, Cunha E, Bica K, Reis S, Saraiva ML. Active pharmaceutical ingredients based on salicylate ionic liquids: insights into the evaluation of pharmaceutical profiles. New Journal of Chemistry. 2013; 37: 4095-4102.

Romeli FJ, Wilfred CD. Synthesis and characterization of flufenamic ionic liquids. Journal of Applied Sciences. 2014; 14(23):33733376 .

Sayeed F, Ahmed A, Sayeed A. Formulation and in vitro evaluation of solid dispersion of fluconazole. International Journal of Pharmaceutical Sciences and Research, 2016; 7(10): 4170-4179.

Sharma R, Kaur G, Kapoor DN. Fluconazole loaded cubosomal vesicles for topical delivery. International Journal of Drug Development and Research, 2015; 7(3): 032-041.

Singh A, Sharma PK, Majumdar DK. Development and validation of different UV- spectrophotometric methods for the estimation of fluconazole in bulk and in solid dosage form. Indian Journal of Chemical Technology, 2011; 18: 357-362.

Stoimenovski J, Macfarlane DR, Bica K, Rogers RD. Crystalline vs ionic liquid salt forms of active pharmaceutical ingredients: A position paper. Pharmaceutical Research,2010; 27(4): 521-526.

Yurtdas, G, Demirel M, Genc L. Inclusion complexes of fluconazole with $\beta$-cyclodextrin: physicochemical characterization and in vitro evaluation of its formulation. Journal of Inclusion Phenomena and Macrocyclic Chemistry. 2011; 70: 429-435.

\section{How to cite this article:}

Mangrule V, Pore Y, Disouza J. Synthesis and physicochemical studies of fluconazole ionic liquids. J App Pharm Sci, 2017; 7 (11): 084-089. 